\title{
Faecal calprotectin concentration in children with coeliac disease
}

\author{
Anna Szaflarska-Popławska ${ }^{1}$, Bartosz Romańczuk ${ }^{2}$, Monika Parzęcka ${ }^{1}$ \\ ${ }^{1}$ Department of Pediatric Endoscopy and Gastrointestinal Function Testing, Collegium Medicum in Bydgoszcz, Nicolaus \\ Copernicus University in Torun, Poland \\ 2Department of Pediatric, Allergology and Gastroenterology, Collegium Medicum in Bydgoszcz, Nicolaus Copernicus Univeristy \\ in Torun, Poland
}

Gastroenterology Rev 2020; 15 (1): 44-47

DOI: https://doi.org/10.5114/pg.2020.93630

Key words: coeliac disease, faecal calprotectin, children, clinical picture.

Address for correspondence: Anna Szaflarska-Popławska, Department of Pediatric Endoscopy and Gastrointestinal Function Testing, Collegium Medicum, Nicolaus Copernicus University, 9 M. Skłodowskie-Curie St, 85-094 Bydgoszcz, Poland, phone: +48 525854850 , e-mail: aszaflarska@wp.pl

\begin{abstract}
Introduction: It is still unknown whether faecal calprotectin elevation may be caused by active untreated coeliac disease $(C D)$ itself or whether it indicates the coexistence of $C D$ and another disease associated with gastrointestinal inflammation.

Aim: To assess faecal calprotectin concentration (FCC) and its correlation with the clinical form and histopathological picture of the small intestine in children with CD.

Material and methods: Fifty-five children with newly recognised CD (mean age: 9.1 years) and 17 children with CD diagnosed at least year before and on a strict gluten-free diet (mean age: 12.3 years) were accepted for the study. Classical $(n=27)$, non-classical $(n=17)$, and asymptomatic form $(n=11)$ were distinguished in children with newly diagnosed CD based on the clinical picture. The histopathological small intestinal lesions were classified as Marsh $1(n=4), 3 a(n=5), 3 b(n=20)$, and $3 c$ $(n=26)$. FCC was assessed using ELISA method with $50 \mu \mathrm{g} / \mathrm{g}$ as the upper limit of the normal.

Results: FCC was abnormal for 21 patients with newly diagnosed CD (38.2\%) and for six patients from the treated group (35.3\%). Mean FCC for the analysed group of patients was $91.7 \pm 144.8 \mu \mathrm{g} / \mathrm{g}$, in the group with newly diagnosed CD $100.9 \pm 154.4 \mu \mathrm{g} / \mathrm{g}$, and in the treated group $-61.8 \pm 106.2 \mu \mathrm{g} / \mathrm{g}(Z=-1.333 ; p=0.183)$. In the group of patients with recently diagnosed CD a statistically significant relationship was not observed for FCC and both clinical picture $\left(\chi^{2}=0.319, p=0.852\right)$ and severity of small intestinal lesions according to the Marsh classification (rho $=0.136$ ).

Conclusions: Assessment of FCC seems to have no use as a marker for diagnostics and monitoring of CD irrespective of clinical form of the disease and severity of the inflammatory lesions within the small intestine.
\end{abstract}

\section{Introduction}

Calprotectin is a $36.5 \mathrm{kDa}$ calcium- and zinc-binding protein, which is mainly exhibited in the cytoplasm of neutrophils. It is also expressed on activated monocytes and macrophages. It can by produced by bone marrow cells, squamous epithelial cells, vascular endothelial cells, and fibroblasts, as a result of activation. It shows antibacterial and chemotactic properties, induces apoptosis, and takes part in interaction between leukocytes and vascular endothelial cells [1].

Many conditions have been linked to an elevation of faecal calprotectin concentration (FCC). However, the current clinical use of faecal calprotectin is focused on distinguishing between inflammatory bowel disease and irritable bowel syndrome and monitoring the activity of inflammatory bowel disease for relapse or postoperative recurrence. The evidence for faecal calprotectin elevation in most other conditions is limited to small observational studies. It is still not known whether faecal calprotectin elevation may be caused by active untreated coeliac disease $(C D)$ itself or whether it indicates the coexistence of $C D$ and another disease associated with gastrointestinal inflammation. The high rate of comorbidity between inflammatory bowel diseases and CD has already been confirmed [2]. A recently published study also provides supporting evidence for a strong association between CD and microscopic colitis [3]. 


\section{Aim}

The aim of this study was to compare FCC in the group of children with isolated recently diagnosed (not treated) $C D$ and in the group of children with $C D$ on a strict gluten-free diet, and to assess the correlation between FCC and both clinical manifestations and severity of small intestinal lesions in patients with the active form of the disease.

\section{Material and methods}

This study was conducted between January 2016 and March 2017. Two groups of children were included. Children with newly diagnosed CD before introduction of a gluten-free diet comprised group A. Group B included children with previously diagnosed $C D$, currently asymptomatic, and declaring strict adherence to the gluten-free diet for at least 1 year. All patients with positive personal or family history concerning inflammatory bowel disease, rheumatological disease, and also on long-term non-steroidal anti-inflammatory therapy were excluded from the study. Self-reported adherence to a gluten-free diet was confirmed by an experienced clinical dietitian at a specific structured dietetic review, and by negative serum tissue transglutaminase antibody.

Based on the clinical picture, group A were classified as patients with classical (with signs and symptoms of malabsorption), non-classical (symptomatic, but without signs and symptoms of malabsorption), and asymptomatic (without symptoms associated with celiac disease) form of the disease.

Coeliac disease diagnosis for all the patients was performed based on the diagnostic criteria of European Society for Paediatric Gastroenterology, Hepatology, and Nutrition (ESPGHAN), which were valid at the diagnosis. Coeliac disease was confirmed histologically for all children based on the evaluation of small intestinal biopsy specimens according to the Marsh-Oberhuber classification. In all IgA-competent patients, simultaneously with the stool examination, IgA anti-tissue transglutaminase antibody (QUANTA Flash tTGIgA, CIA, Inova Diagnostics, Inc.) was determined. In IgA-deficient patients IgG anti-tissue transglutaminase antibody (QUANTA Flash tTGIgG, CIA, Inova Diagnostics, Inc.) was assessed.

Faecal calprotectin concentration was performed in a specialised laboratory using sandwich ELISA assay with two types of mouse monoclonal antibody directed against human calprotectin (Quantum Blue ${ }^{\oplus}$ Calprotectin Extended, Bühlmann). This assay enables quantitative calprotectin determination in the 30 to $1000 \mu \mathrm{g} / \mathrm{g}$ range. $50 \mu \mathrm{g} / \mathrm{g}$ was accepted as the upper limit of normal FCC.

The study was approved by the Local Bioethics Committee.

\section{Statistical analysis}

The statistical software IBM SPSS 24.0 was used for statistical analysis. Kolmogorov-Smirnov test was used to assess the equality of probability distributions. Non-parametric Mann-Whitney $U$ test was used for distribution other than normal.

\section{Results}

Group A consisted of 55 children, one to 18 years old (mean age: 9.1 years), including 32 girls and 23 boys with newly diagnosed, untreated CD. In this group, 27 children were diagnosed with classical form, 17 with non-classical form, and 11 with asymptomatic form of CD. Four patients had Marsh grade 1 histology, 51 children had Marsh grade 3, including 5 children with Marsh grade 3a histology, 20 children with Marsh grade $3 b$ histology, and 26 children with Marsh grade 3c histology.

Group B consisted of 17 patients, 3 to 18 years old (mean age: 12.3 years), including 6 girls and 11 boys with $C D$, diagnosed at least 1 year before, who declared strict adherence to a gluten-free diet.

Faecal calprotectin concentration was abnormal in 21 patients from group $A(38.2 \%)$ and in 6 patients from group B (35.3\%). Mean calprotectin concentration in group of studied patients was $91.7 \pm 144.8 \mu \mathrm{g} / \mathrm{g}$, including $100.9 \pm 154.4 \mu \mathrm{g} / \mathrm{g}$ in group $\mathrm{A}$ and $61.8 \pm 106.2 \mu \mathrm{g} / \mathrm{g}$ in group B. No statistically significant difference was found in the range of mean FCC for group $A$ and $B(Z=$ $-1.333 ; p=0.183$ ) (Figure 1).

Mean calprotectin concentration in children with classical form of CD was $67.9 \pm 84.6 \mu \mathrm{g} / \mathrm{g}$, with non-classical form of the disease it was $155.5 \pm 222.8 \mu \mathrm{g} / \mathrm{g}$, and with asymptomatic form of the disease it was $97.8 \pm 150.7 \mu \mathrm{g} / \mathrm{g}$.

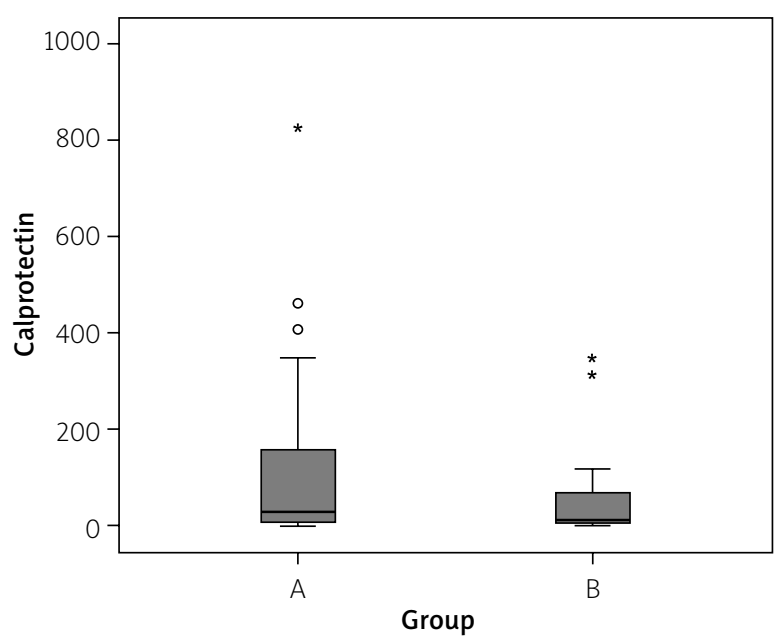

Figure 1. Faecal calprotectin concentration in group $A$ and $B$ 


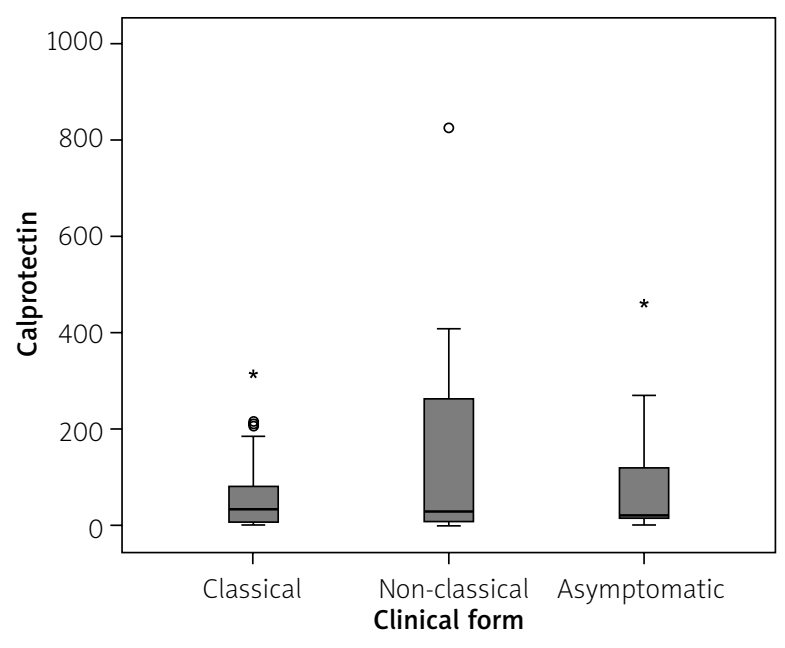

Figure 2. Assessment of the relationship between calprotectin concentration and clinical form of coeliac disease in group $A$

The relationship between calprotectin concentration and clinical form of active CD was analysed using Kruskal-Wallis test, and no statistically significant relationship was observed $(\chi 2=0.319, p=0.852)$ (Figure 2).

The relationship between calprotectin concentration and severity of small intestinal lesions according to Marsh classification was assessed using Spearman correlation, which showed no statistically significant relationship (rho $=0.136, p=0.322$ ).

\section{Discussion}

Coeliac disease is an autoimmune enteropathy triggered by gluten-containing grains in genetically predisposed individuals. Small intestinal biopsy is still accepted as the diagnostic gold standard and is currently used to confirm the diagnosis of CD in the vast majority of patients. Major features of small intestinal lesions suggestive of CD are not only villous atrophy, crypt hyperplasia, and increased intraepithelial lymphocytes but also the influx of inflammatory cells other than lymphocytes in the lamina propria [4].

Normal small intestinal mucosa contains plasmocytes and T lymphocytes, as well as macrophages, and exceptionally eosinophils, but does not include neutrophils, which are a main source of calprotectin. Coeliac disease leads to the migration of inflammatory cells to the mucosal lamina propria of small intestine, including influx of mast cells, neutrophils, and eosinophils in different numbers up to dense cellular infiltrates [4].

The present study attempted to compare FCC in patients with active, newly diagnosed CD and in patients with non-active disease, treated for at least 1 year with a gluten-free diet. Moreover, it aimed to assess the cor- relation between FCC and both clinical form of the disease and severity of mucosal lesions in patients with active $C D$.

The mean FCC was higher in patients with untreated CD in comparison to patients with treated CD (100.9 vs. $61.7 \mu \mathrm{g} / \mathrm{g}$ ), but this difference was statistically insignificant $(p=0.183)$. Additionally, the relationship between FCC and the form of the disease (classical vs. non-classical vs. asymptomatic CD), as well as the severity of mucosal damage in small intestine according to the Marsh classification, were not confirmed.

Only a few studies that determine the utility of FCC assessment in coeliac patients have been published in the literature, and their results are not consistent. In two studies conducted with adult patients (28 and 50 patients) no statistically significant difference was observed in FCC between patients with recently diagnosed $C D$ and the control group $[5,6]$. On the other hand, studies concerning paediatric patients show that assessment of FCC can be accepted as a useful marker of intestinal inflammation in CD, especially in patients with gastroenterological manifestation. Ertekin et al. [7] stated that the FCC in a group of 29 children with recently diagnosed CD was significantly higher in comparison with a group of healthy children $(p=0.004)$ and in comparison with children on a gluten-free diet for at least 1 year $(p=0.001)$. Similarly to the aforementioned study, Balamtekin et al. [8] confirmed significantly higher FCC in untreated children with CD in comparison with treated children and with the control group $(p<0.001)$. In a group of 63 children with recently diagnosed CD, studied by Biskou et al. [9], patients with CD had higher FCC than their healthy peers (36.4 mg/kg vs. $25 \mathrm{mg} / \mathrm{kg}$ ).

Explanation of the differences in the determination of FCC as a marker of intestinal inflammation in paediatric and adult CD is difficult. Reports in the literature emphasise that FCC can vary in different age groups. FCC in infants is higher and drops to the level observed in adults in the fifth year of life [3]. In studies by Ertekin et al. [7] and Balamtekin et al. [8] the mean age of children with recently diagnosed CD was slightly higher than 6 years, compared with 9.1 years in our own study. The age of patients on a gluten-free diet was also lower in Balametkin's studies [8] in comparison to the author's own research (12.3 vs. 10.1 years old). Adoption of a similar cut-off point for normal calprotectin concentration irrespective of the age of studied patients can explain the high percentage of both treated and untreated children with abnormal results in the author's own study (37.5\% for $50 \mu \mathrm{g} / \mathrm{g}$ cut-off point); however, it does not explain the differences in FCC in the comparison of healthy or affected children matched in terms of gender and age. On the other hand, lowering of FCC ob- 
served in paediatric patients on a gluten-free diet [7-9] can be a natural decreasing tendency related to the adolescence of patients and not an effect of a gluten-free diet. Lower FCC in treated patients in comparison with untreated patients was also observed in the author's own study, but differences were statistically insignificant. Regardless of the differences in the results of studies, attention should be paid to the small number groups both in paediatric studies ( $\leq 63$ children) and in adult studies ( $\leq 50$ patients).

Comparison of author's own study results and results of other studies concerning correlation between FCC and both the clinical and histopathological picture in groups of patients with active CD is particularly difficult. Correlation of FCC with the clinical picture of CD was assessed in four studies (positive correlation was confirmed by Balamtekin et al. [8]; lack of correlation was shown in studies of Montalto et al. [5], Capone et al. [6], and in the author's own studies). Correlation of FCC with severity of the intestinal inflammatory lesions was assessed in five studies (increased FCC in children with flat mucosa in comparison with children with partial villous atrophy $(p=0.001)$ in a study by Ertekin et al. [7]; lack of correlation in other studies [5, $6,8]$ and in the author's own study). These discrepancies are presumably the result of the small groups of patients.

A limited number of publications have focused on the use of faecal calprotectin measurements in the diagnosis and monitoring of patients with CD, and their contradictory results show that any final conclusion should be drawn with caution, which also indicates the direction of investigation for further studies. However, the lack of a statistically significant relationship between FCC and both clinical and histopathological activity of newly diagnosed paediatric CD in our own study suggests the uselessness of FCC assessment for CD diagnosis. Supposing that the majority of children within 1 year of a strict gluten-free diet fully recover from the mucosal damage inflicted by CD, a lack of statistically significant differences in FCC between active and non-active CD excludes its role in predicting of microscopic recovery and its usefulness for CD follow-up.

\section{Conflict of interest}

The authors declare no conflict of interest.

\section{References}

1. Vaos G, Kostakis ID, Zavras N, et al. The role of calprotectin in pediatric disease. Biomed Res Intern 2013; 2013: 542363.

2. Shah A, Walker M, Burger D, et al. Link between celiac disease and inflammatory bowel disease. J Clin Gastroenterol 2019; 53: 514-22.
3. Sonnenberg A, Turner KO, Genta RM. Associations of microscopic colitis with other lymphocytic disorders of the gastrointestinal tract. Clin Gastroenterol Hepatol 2018; 16: 1762-7.

4. Oberhuber G, Granditsch G, Vogelsang H. The histopathology of coeliac disease: time for a standarized report scheme for pathologists. Eur J Gastroenterol Hepatol 1999; 11: 1185-94.

5. Montalto M, Santoro L, Curigliano V, et al. Faecal calprotectin concentrations in untreated coeliac disease patients. Scan J Gastroenterol 2007; 42: 957-61.

6. Capone P, Rispo A, Imperatore $\mathrm{N}$, et al. Fecal calprotectin in coeliac disease. World J Gastroenterol 2014; 20: 611-2.

7. Ertekin V, Selimo lu MA, Turgut A, et al. Fecal calprotectin concentration in celiac disease. J Clin Gastroenterol 2010; 44: 544-6.

8. Balamtekin N, Baysoy G, Uslu N, et al. Fecal calprotectin concentration is increased in children with celiac disease: relations with histopathological findings. Turk J Gastroenterol 2012; 23: 503-8.

9. Biskou O, Gardner-Medwin J, Mackinder M, et al. Faecal calprotectin in treated and untreated children with coeliac disease and juvenile idiopathic arthritis. J Pediatr Gastroenterol Nutr 2016; 63: e112-5.

Received: 27.02 .2019

Accepted: 2.05 .2019 